\title{
Comparison of Pyroelectric and Thermopile Detectors
}

\author{
Norbert Neumann ${ }^{1}$, Victor Banta ${ }^{2}$ \\ ${ }^{1}$ InfraTec GmbH, Gostritzer Str. 61-63, 01217 Dresden, Germany \\ n.neumann@infratec.de \\ ${ }^{2}$ Dexter Research Center, Inc., 7300 Huron River Drive, Dexter, MI 48130, USA
}

\begin{abstract}
:
Thermal infrared detectors are distinguished by the advantages of a wide wavelength response, no requirement for cooling, high-temperature stability, high signal-to-noise ratio and low cost. Consequently, they are widely used in consumer products and in instrumentation. In the literature one can find many publications with regard to details either of pyroelectric detectors or thermopiles but a comparison of both thermal detectors could seldom be found. The main principles and the basic design of both the pyroelectric detector and the thermopile will be discussed as a starting point. Then the electro-optical properties of typical representatives of both the pyroelectric detector and the thermopile produced from our companies will be compared. Measurement results of blackbody responsivity, noise, and specific detectivity and spectral response will be displayed and discussed. The authors will conclude with a discussion of the advantages and disadvantages of pyroelectric detectors and thermopiles, including the electro-optical properties as well as the costs and typical applications of both thermal detector types.
\end{abstract}

Key words: Pyroelectric Detector, Thermopile Detector, Responsivity, Specific Detectivity.

\section{Introduction}

Thermal infrared detectors are distinguished by the advantages of a wide wavelength response, no requirement for cooling, high-temperature stability, high signal-to-noise ratio and low cost. Consequently, they are widely used in medical, industrial, military and consumer products [1]. Publications exist with information about pyroelectric or thermopile detectors, but direct comparisons of the two detectors is rarely found. Therefore we decided to compare pyroelectric detectors and thermopiles and chose two typical representatives from the production portfolio of our companies.

\section{Principles and Basic Design of Pyroelectric and Thermopile Detectors}

Both the pyroelectric and the thermopile detectors are thermal detectors. The thermal conversion is the basis for a high responsivity and signal-to-noise ratio and should result in a high temperature change $\Delta T_{S}$ of the respective radiation sensitive element. Figure 1 represents a simplified thermal model [2]. The radiation sensitive element is characterized by the absorption rate $\alpha$, the thickness $t_{P}$, the area $A_{S}$, the heat capacity $H_{P}$ and the thermal conductance $G_{T}$ to its surroundings which is represented by a heat sink with a given temperature $T_{A}$.

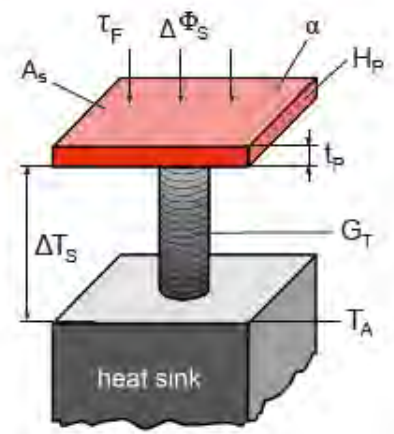

Fig. 1. Simplified thermal model of pyroelectric and thermopile detectors

Using the thermal time constant

$$
\tau_{T}=\frac{H_{P}}{G_{T}}
$$

the temperature difference results in

$$
\Delta T_{P}=\frac{\alpha \Phi_{S}}{\sqrt{G_{T}^{2}+\omega H_{P}^{2}}}
$$

or for sinusoidal agitation in the steady state

$$
\Delta \widetilde{T}_{P}=\frac{\alpha \widetilde{\Phi}_{S}}{G_{T}} \cdot \frac{1}{\sqrt{1+\tau_{T}^{2}}}
$$


For significant temperature differences to occur the absorbance $\alpha$ has to be as near to $100 \%$ as possible. This can especially be achieved by the use of a special absorption layer. The heat capacity value $\mathrm{H}_{\mathrm{P}}$ has to be low. Compromises are necessary as the required reduction in the thermal conductance $G_{T}$ is opposed by the increase of the thermal time constant $\tau_{\mathrm{T}}$.

The thermal to electrical conversion is different in pyroelectric and thermopile detectors. In pyroelectric detectors the thermal to electrical conversion is due to the pyroelectric effect $p$. A very thin pyroelectric plate with top and bottom electrodes forms the radiation sensitive detector element. Changing the temperature by incident radiation of the pyroelectric plate will influence charges in the electrodes. The resulting short circuit current is proportional to the temperature rate:

$$
i_{P}=p A_{S} \frac{\Delta T_{P}}{d t}
$$

This pyroelectric current, supplied by a highimpedance source has to be converted by a preamplifier with a high-impedance input. There are two alternatives available: voltage mode and current mode. The voltage mode can be implemented using a voltage follower and the current mode using an inverting operational amplifier (Op Amp) as seen in figure 2 [3].
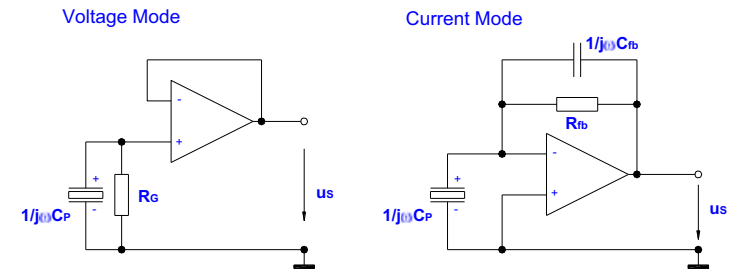

Fig. 2. Alternative preamplifier modes of pyroelectric detectors [4].

The thermopile detector is an array of $n$ miniature thermocouple junctions connected in series as differential pairs. These differential pairs make up the cold junctions and the hot junctions (see figure 4). In fact, the hot and cold junctions are connected by two dissimilar materials with a large thermoelectric power and opposite polarities, called "Arms", creating a Seebeck effect between the junctions. A voltage is produced, proportional to the temperature gradient between the hot and cold junctions [5].

$$
V_{t h}=n\left(\alpha_{A}-\alpha_{B}\right) \Delta T_{S}
$$

For thin film based thermopiles, the arm materials are antimony ( $\mathrm{Sb}$ ) and bismuth $(\mathrm{Bi})$. For Silicon thermopiles, the arm materials can be alternating n-type and p-type Poly-Silicon or n-type with gold $(\mathrm{Au})$ or aluminum $(\mathrm{Al})$. The cold junctions are typically thermally connected to the detector package and are located around the perimeter of the substrate opening. The hot junctions are located in the center of the detector pattern and are coated with an energy absorber. The hot junctions define the active area of the detector and are suspended on a thin membrane, thermally isolating them from the rest of the package.

Fig. 3 depicts graphically the frequency dependence of the temperature change, the pyroelectric short circuit current and the open circuit thermopile voltage of a pyroelectric and a thermopile detector, respectively. Assuming a thermal time constant of about $150 \mathrm{~ms}$ the temperature change of the radiation sensitive element achieves a saturation value below the corner frequency of about $1 \mathrm{~Hz}$ and has a rolloff $20 \mathrm{~dB} /$ decade above. Whereas the open circuit voltage (signal) of a thermopile behaves in the same manner the short circuit pyroelectric is proportional to the derivative $\Delta \mathrm{T}_{\mathrm{S}} / \mathrm{dt}$. The pyroelectric current increases with the frequency with $20 \mathrm{~dB} /$ decade and achieves a saturation value above the thermal corner frequency.

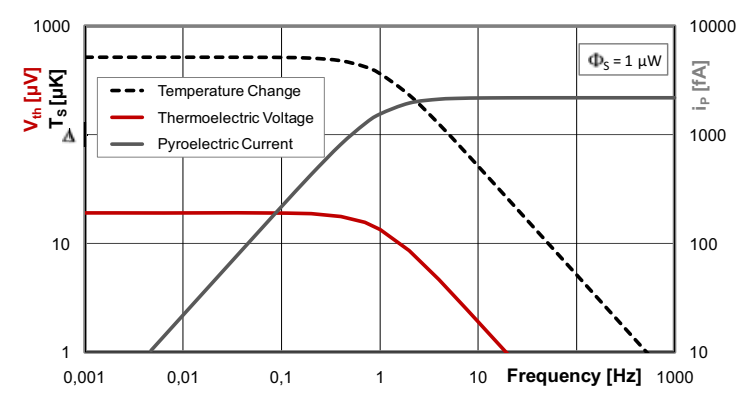

Fig. 3. Frequency dependence of the temperature change of a radiation sensitive element, of the pyroelectric short-circuit current and of the opencircuit thermopile voltage.

In table 1 the principles, basic design and samples of both pyroelectric and thermopile detectors are summarized.

\section{Electro-Optical Properties}

For the comparison of the electro-optical properties typical representatives of both the pyroelectric detector and the thermopile with a sensitive area of $1.5 \times 1.5$ and $2 \times 2 \mathrm{~mm}^{2}$ and $\mathrm{BaF}_{2}$ window were chosen. LME-302 and LME335 are $\mathrm{LiTaO}_{3}$ based pyroelectric detectors working in voltage and current mode, respectively. ST150 and $2 \mathrm{M}$ are $\mathrm{Bi} / \mathrm{Sb}$ thin film based and poly-silicon based thermopile detectors filled with dried nitrogen and argon, respectively. 
Table. 1: Summary of the comparison of both pyroelectric and thermopile detectors

\begin{tabular}{|c|c|c|}
\hline Transducer & Pyroelectric Detector & Thermopile Detector \\
\hline Conversion Effect & Pyroelectric effect & Seebeck effect \\
\hline Signal & Short-circuit current & Open-circuit voltage \\
\hline Responsivity & Proportional to $\Delta \mathrm{T}_{\mathrm{S}} / \mathrm{dt}$ & Proportional to $\Delta \mathrm{T}_{\mathrm{S}}$ \\
\hline Basic Design & & \\
\hline Example & & \\
& &
\end{tabular}

For the comparison the responsivity and the noise density were measured at $23^{\circ} \mathrm{C}$ in a broad frequency range of $0.1 \mathrm{~Hz}-4.2 \mathrm{kHz}$ and $0.76 \mathrm{~Hz}-11.7 \mathrm{kHz}$, respectively.

For the measurement of the absolute responsivity at a modulation frequency of $10 \mathrm{~Hz}$ and a blackbody temperature of $500 \mathrm{~K}$ the model 563/301 from Infrared Systems Development Corporation was used. Choosing a blackbody to detector distance of about $117 \mathrm{~nm}$ an irradiance of about $7 \mu \mathrm{W} / \mathrm{mm}^{2}$ was achieved.

The relative responsivity is measured with a high-stability super luminescent emitting diode (DenseLight DL-BZ1-CS65M5A) at a wavelength of $1635-1665 \mathrm{~nm}$. The diode is modulated in a so-called multi-sinusoidal mode with frequencies between $0.1 \mathrm{~Hz}$ and $4.2 \mathrm{kHz}$.

The noise of the detectors at $23^{\circ} \mathrm{C}$ is measured with the $24 \mathrm{bit} / 204.8 \mathrm{kS} / \mathrm{s}$ 4-Input Dynamic Signal Analyzer PXI 4462 and a PXI-1042Q chassis from National Instruments. The noise density is then calculated by a software based Fourier transformation. Especially for low noise detectors an ultra-low noise preamplifier Model 5184 from Signal Recovery with a $30 \mathrm{~dB}$ gain was additionally used. The measured noise density (see Fig. 5) of a $10 \Omega$ resistor at the input of the model 5184 illustrates the ultra-low noise of the preamplifier. Only at very low frequencies one can obtain an increase of the noise voltage.

In Fig. 4 the frequency dependence of the responsivity of pyroelectric and thermopile detectors is shown.

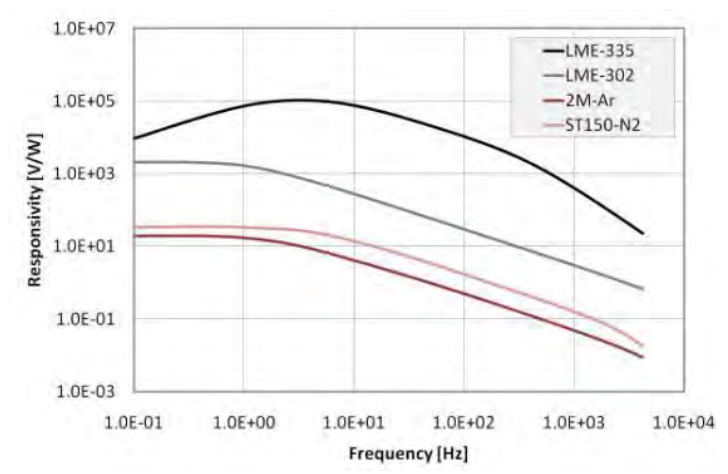

Fig. 4. Frequency dependence of the responsivity of pyroelectric (LME-302, LME-335) and thermopile detectors (ST150, 2M).

The thermopile detectors exhibit an inherently stable response to $\mathrm{DC}$ radiation in contrast to pyroelectric detectors. Above the corner frequency which is determined from the thermal time constants of $85 \mathrm{~ms}$ and $38 \mathrm{~ms}$ of the $2 \mathrm{M}$ and ST150 thermopile detectors, respectively, the response of the thermopile detectors is reduced by $20 \mathrm{db} /$ decade. Pyroelectric detectors feature a much higher responsivity but are characterized, in contrast to thermopile detectors, by two time constants. In the case of the voltage mode pyroelectric detector LME302 the thermal time constant of 150 ms results in a corner frequency of about $1 \mathrm{~Hz}$ and a $20 \mathrm{db} /$ decade roll-off of the responsivity at frequencies above the corner frequencies. The electrical time constant of about $5 \mathrm{~s}$ results in a roll-off of the responsivity at frequencies lower than $32 \mathrm{mHz}$ but is not within measured frequency range. The current mode detector is characterized by the same thermal time constant of $150 \mathrm{~ms}$ but features a much lower electrical time constant of $20 \mathrm{~ms}$ resulting in 
corner frequencies of $1 \mathrm{~Hz}$ and $8 \mathrm{~Hz}$, respectively.

The absorption layer on top of the radiation sensitive elements causes an additional roll-off of the response in the $\mathrm{kHz}$ range. The higher the thermal resistance of the absorption layer the lower the roll-off frequency which could be clearly noticed for LME-335 and ST150.

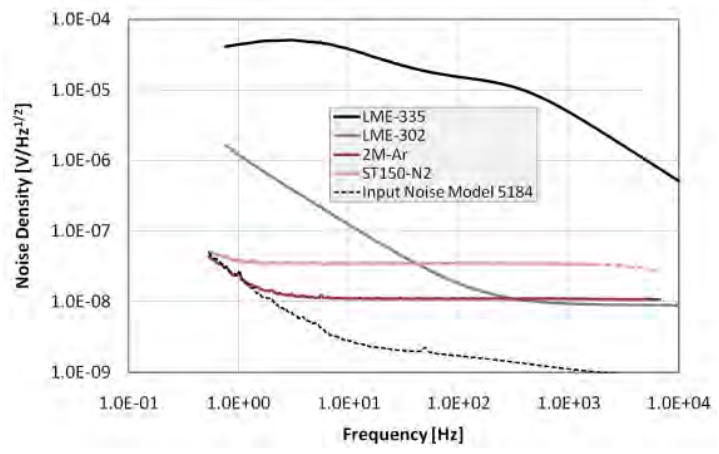

Fig. 5. Frequency dependence of the noise density of pyroelectric (LME-302, LME-335) and thermopile detectors (ST150, 2M).

In Fig. 5 the noise density of pyroelectric and thermopile detectors are compared. The thermopile detectors are distinguished by a low Johnson noise $v_{n}$ in a broad frequency range cause by the internal thermopile resistance $R_{\text {th }}$,

$$
v_{n}=\sqrt{4 k T R_{t h} B}
$$

where $\mathrm{k}$ is the Boltzmann constant, $\mathrm{T}$ the absolute temperature and $B$ the noise bandwidth. In the frequency range below several $\mathrm{Hz}$ the noise density of the preamplifier dominates the measured noise and one can assume that the noise of the thermopile is flat also until a frequency of at least $1 \mathrm{~Hz}$. The argon filled thermopile $2 \mathrm{M}$ shows a decrease of the noise density at frequencies above about several $\mathrm{kHz}$ because of the high internal resistance of $77 \mathrm{k} \Omega$ and a significant cable capacitance.

The noise density of pyroelectric detectors is dominated from different noise sources. At low frequencies until several $10 \mathrm{~Hz}$ Johnson noise of the high meg-ohm resistor, the current noise of the preamplifier and the temperature fluctuation noise are the dominant noise sources. In the middle frequency range until about $1 \mathrm{kHz}$ the dielectric loss of the pyroelectric material dominates the noise density. Above $1 \mathrm{kHz}$ the voltage noise of the preamplifier is the dominant noise source. Although the same noise sources are dominant in the same frequency ranges the frequency dependence in voltage and current mode is different due to the different preamplification of signal and noise and the different electrical time constants.

In Fig. 6 the calculated specific detectivity $D^{*}$ of pyroelectric and thermopile detectors are compared.

$$
D^{*}=\sqrt{A_{S} R_{V} / v_{n}}
$$

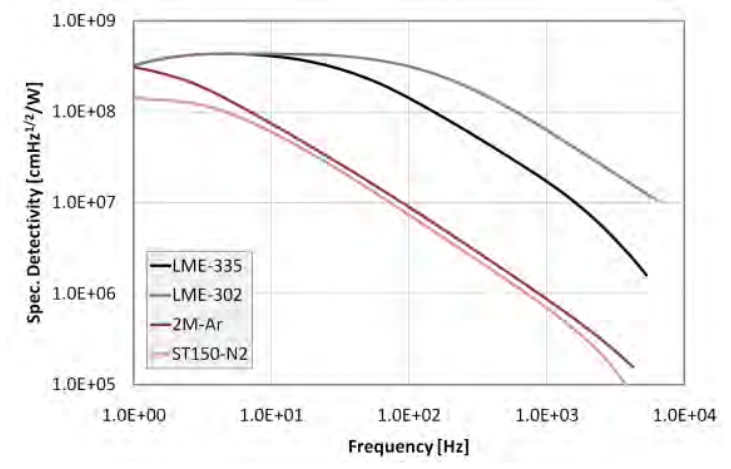

Fig. 6. Frequency dependence of the specific detectivity of pyroelectric (LME-302, LME-335) and thermopile detectors (ST150, 2M).

The pyroelectric detectors features a high specific detectivity of $3-4 \cdot 10^{8} \mathrm{cmHz}^{1 / 2} / \mathrm{W}$ in a very broad frequency range of $1-100 \mathrm{~Hz}$. Only argon filled $\mathrm{Bi}-\mathrm{Sb}$ thin film based thermopiles receive a specific detectivity in the order of $3-4 \cdot 10^{8} \mathrm{cmHz}^{1 / 2} \mathrm{~W}$ at very low frequencies of $1 \mathrm{~Hz}$ and below. The specific detectivity of silicon based thermopile detectors is lower than the $\mathrm{Bi}-\mathrm{Sb}$ thin film based, especially below a frequency of $10 \mathrm{~Hz}$. The reason for different behavior above about $10 \mathrm{~Hz}$ of the pyroelectric detectors LME-302 and LME-335 is based on the preamplifier voltage noise and the doubled input capacitance of the parallel compensated LME-335. The voltage noise of the JFET in the voltage mode source follower (LME-302) is lower than the voltage noise of the CMOS Op Amp of the current mode trans-impedance amplifier (LME-335).

\section{Summary and Discussion}

Pyroelectric and thermopile detectors are both thermal infrared transducers. Therefore responsivity and specific detectivity are high especially at low frequencies.

Thermopile detectors can be used without any chopper to detect infrared DC radiation. Further advantages are low noise and the absence of a microphonic effect. Thermopile detectors are often combined with chopper stabilized amplifiers in order to overcome the disadvantage of a very low noise voltage. As a result of their low cost thermopile detectors were applied in simple gas analysis, noncontact temperature measurements, and fire detection. 
Pyroelectric detectors are distinguished by a much higher (20-40 dB) responsivity and also a higher specific detectivity. Special care must be taken for the microphonic effect. It could be reduced by a special chip mounting to a certain degree but it does not completely disappear [6]. Pyroelectric detectors are used in high performance gas analyzers, flame detection devices and scientific instrumentation. In table 2 the parameters, advantages and disadvantages of both pyroelectric and thermopile detectors are summarized.

Table. 2: Parameters, advantages and disadvantages of both pyroelectric and thermoelectric detectors

\begin{tabular}{|c|c|c|c|c|c|}
\hline & & \multicolumn{2}{|c|}{ Thermopile } & \multicolumn{2}{|c|}{ Pyroelectric Detector } \\
\hline Parameter & & $2 \mathrm{M}$ & ST150 & LME-335 & LME-302 \\
\hline Type & & $\begin{array}{c}\text { thin film based } \\
\text { Bi-Sb }\end{array}$ & $\begin{array}{c}\text { silicon based } \\
\text { poly-silicon }\end{array}$ & $\begin{array}{c}\mathrm{LiTaO}_{3}, \text { Current } \\
\text { Mode, } \\
\text { compensated }\end{array}$ & $\begin{array}{c}\mathrm{LiTaO}_{3} \\
\text { Voltage Mode }\end{array}$ \\
\hline Window & & $\mathrm{BaF}_{2}$ & $\mathrm{BaF}_{2}$ & $\mathrm{BaF}_{2}$ & $\mathrm{BaF}_{2}$ \\
\hline Active Area Size & $\mathrm{mm}^{2}$ & $2.0 \times 2.0$ & $1.5 \times 1.5$ & $2.0 \times 2.0$ & $2.0 \times 2.0$ \\
\hline Thermal Time Constant & $\mathrm{ms}$ & 85 & 38 & 150 & 150 \\
\hline Electrical Time Constant & $\mathrm{ms}$ & - & - & 20 & 4700 \\
\hline $\begin{array}{c}\text { Responsivity } \\
\left(500 \mathrm{~K}, 10 \mathrm{~Hz}, 25^{\circ} \mathrm{C}\right)\end{array}$ & VM & 4,1 & 13,9 & 77800 & 274 \\
\hline $\begin{array}{c}\text { Responsivity } \\
\left(500 \mathrm{~K}, \mathrm{DC}, 25^{\circ} \mathrm{C}\right)\end{array}$ & VM & 19 & 34 & - & - \\
\hline $\begin{array}{c}\text { Temperature Coefficient of } \\
\text { Responsivity }\end{array}$ & $\mathrm{ppm} / \mathrm{K}$ & -3600 & -400 & 1000 & 200 \\
\hline $\begin{array}{c}\text { Noise Density } \\
\left(10 \mathrm{~Hz}, \mathrm{BW} 1 \mathrm{~Hz}, 25^{\circ} \mathrm{C}\right)\end{array}$ & $\mathrm{nVHz} z^{-1 / 2}$ & 11,0 & 34,8 & 3810 & 12,6 \\
\hline $\begin{array}{c}\text { Spec. Detectivity } \\
\left(500 \mathrm{~K}, 10 \mathrm{~Hz}, 25^{\circ} \mathrm{C}\right)\end{array}$ & $10^{8} \mathrm{cmHz}^{-1 / 2} \mathrm{~W}^{-1}$ & 0,8 & 0,6 & 4,1 & 4,4 \\
\hline $\begin{array}{l}\text { Spec. Detectivity } \\
\left(500 \mathrm{~K}, \mathrm{DC}, 25^{\circ} \mathrm{C}\right)\end{array}$ & $10^{8} \mathrm{cmHz}^{-1 / 2} \mathrm{~W}^{-1}$ & 3,5 & 2,0 & & \\
\hline \multicolumn{2}{|l|}{ Advantage } & \multicolumn{2}{|c|}{$\begin{array}{c}\mathrm{DC} \& \mathrm{AC} \text { response, no } \\
\text { biasing, low cost }\end{array}$} & \multicolumn{2}{|c|}{ high responsivity, high SNR } \\
\hline \multicolumn{2}{|c|}{ Disadvantage } & \multicolumn{2}{|c|}{ low responsivity } & \multicolumn{2}{|c|}{ vibration responsive } \\
\hline
\end{tabular}

\section{References}

[1] H. Budzier, G. Gerlach, Thermal infrared sensors: theory, optimization and practice, John Wiley \& Sons Ltd, United Kindom, 2011. ISBN 978-0-470-87192-8

[2] E. H. Putley, The pyroelectric detector, Semiconductors and Semimetals, Vol. 5, Infrared Detectors, R. K. Willardson and A. C. Beer (Eds.), Academic press, New York, 1970

[3] S. G. Porter, A brief guide to pyroelectric detectors, Ferroelectrics $33(1 / 2 / 3 / 4)$, 193206 (1980)

[4] Application Note InfraTec $\mathrm{GmbH}$, Advanced features of InfraTec pyroelectric detectors, http://www.infratec.de/en/sensordivision/literature/application-notes.html

[5] Application Briefs Dexter Research Center, Inc., Introduction to Thermopile Detectors, http://www.dexterresearch.com/?module=P age\&sID=technical-library

[6] N. Neumann, H. Sänze, How to reduce the microphonic effect in pyroelectric detectors?, IRS² Conference 2008, May 6-7, 2008 